postmenopausal women at higher risk for fracture whereas they display a moderately reduced aBMD. Hip QCT and HRpQCT may also improve the prediction for fracture, but they are still research techniques.

Disclosure of Interest: None declared

DOI: 10.1136/annrheumdis-2017-eular.7232

\section{SP0027 EMERGING THERAPIES FOR OSTEOPOROSIS}

\section{P. Geusens. Rheumatology, MaastrichtUMC and UHasselt, Genk, Netherlands}

Currently, anti-resorptive drug therapy is the cornerstone of fracture prevention. Anti-resorptive drugs decrease bone remodelling, allowing the remaining bone to increase secondary mineralisation, and, with denosumab, also allowing periosteal bone modelling.

Teriparatide is currently the only available bone-forming drug, which increases bone formation more than bone resorption, and has been shown to reduce the risk of vertebral and non-vertebral fractures.

Recently, two randomized clinical trials have been published on the effect of new bone forming agents on the risk of fracture.

Abaloparatide is a 1-34 fragment of PTHrP, with different pharmacokinetics and potential different signalling mechanisms compared to teriparatide. It increases bone formation more than bone resorption, both to a lesser degree than teriparatide. In the double blind, randomised placebo-controlled 18-month ACTIVE study, abaloparatide significantly reduced the risk of vertebral $(-86 \%)$, clinical $(-43 \%)$, non-vertebral $(-43 \%)$ and major fractures $(-70 \%)$. In a parallel randomised exploratory open-label comparison with teriparatide, bone density increased significantly more with abaloparatide, but the anti-fracture effect was similar, except for a significantly better result on prevention of major fractures.

Romosozumab is a monoclonal antibody that binds sclerostin, which is an inhibitor of bone formation. In contrast to other bone forming agents, it disconnects the increase in bone formation from a decrease in bone resorption. In the double-blind placebo-controlled FRAME study, romosozumab significantly reduced the risk of vertebral $(-63 \%)$ and clinical fractures $(-36 \%)$ during the first year, an effect that was maintained by transitioning to one-year denosumab treatment.

The effect on non-vertebral fractures was not significant, but geographic interaction was found. When excluding patients from South America, in whom fracture risk was low, one-year treatment with romosozumab significantly decreased the risk of non-vertebral fractures by $42 \%$.

Abaloparatide and romosozumab are new bone forming agents, with different effects on bone remodelling and remodelling and early effects on clinical fractures. This opens new perspectives in individualised treatment of patients with high fracture risk and for fracture prevention in patients with low bone turnover, multiple vertebral fractures, very low BMD and fractures or bone loss during anti-resorptive treatment.

Disclosure of Interest: P. Geusens Grant/research support from: Pfizer, Abbott, Lilly, Amgen, MSD, Will, Roche, UCB, BMS, Novartis, Consultant for: Amgen DOI: 10.1136/annrheumdis-2017-eular.7265

\section{WEDNESDAY, 14 JUNE 2017}

\section{Systematic literature review: the link from science to clinical practice}

\section{SP0028 SYSTEMATIC LITERATURE REVIEW: WHERE TO START}

S. Ramiro. Rheumatology, Leiden University Medical Center, Leiden, Netherlands

Starting a systematic literature review (SLR), particularly for the first time, may be an overwhelming task. As its designation indicates, it requires a systematic approach, in order to ensure that all the relevant literature addressing the research question at hand is covered and appropriately summarized.

SLRs are particularly helpful for clinicians, who have a question based on their clinical practice and want to obtain the best evidence based answer. The large and increasing amount of existing literature makes the life of a clinician difficult when trying to search by him/herself for the answer based on the original studies. An SLR may thus be an efficient way to get the answer, by condensing hundreds of studies into a good summary. They are also of the basis, together with expert opinion, of diagnostic and/or management/treatment recommendations.

The first step of an SLR is always to properly formulate the research question, in a way that the literature covering the topic can be searched. The classic approach is the PICO framework, in which the formulated question includes the different elements guiding the literature search, namely the Population of interest, the Intervention to which patients are exposed, the Comparator against which the effect of the intervention is to be compared and the Outcomes on which the assessment of the intervention will rely and in which the SLR will focus (e.g. efficacy, safety outcomes, etc).

A crucial aspect when reviewing the literature is the risk of bias assessment. Studies should be judged for their methodological quality so that one can interpret their findings accordingly. If a study is methodologically not sound, then we know that it will not be very helpful in answering the research question and, most important, this cannot be ignored when performing an SLR. This risk of bias assessment also requires some knowledge of important epidemiology and biostatistics concepts, which are needed to correctly understand how a study is conducted.

These and other steps contribute to shaping an SLR. Challenges involved when performing an SLR will be discussed in detail in this presentation, as well as the different steps of an SLR and the most critical aspects that should be considered. Tips from experiences with SLRs will be shared. This should ultimately encourage (young) clinicians and researchers on where to start when embarking on an SLR challenge, but hopefully also on how proceed with and bring to an end a successful and informative SLR.

Disclosure of Interest: None declared

DOI: 10.1136/annrheumdis-2017-eular.7140

\section{SP0029 LINKING SCIENCE TO CLINICAL PRACTICE: FROM THE SYSTEMATIC LITERATURE REVIEW TO THE FORMULATION OF RECOMMENDATIONS}

R. Landewe. Rheumatology \& clinical immunology, Amsterdam Rheumatology \& immunology center, Amsterdam, Netherlands

Systematic literature review (SLR) is a scientific method to collect the available data in the literature regarding a specific research question, and to compare the quality of different studies, in order to arrive at the best possible answer. SLR is not the same as data-pooling. In contrast to what many clinicians think, SLR is clinical science and not "easy going", it takes a lot of time and effort, you can make mistakes, and you need to practice and obtain experience. A proper SLR is all but "low hanging fruit".

Nevertheless, SLRs from the evidence base of guidelines, and as such are integral parts of them.

This lecture will describe how SLRs should be translated into guidelines, and what pitfalls may prevent that the appropriate evidence will be enclosed in recommendations, or that too weak evidence gets a too prominent place.

Disclosure of Interest: None declared DOI: 10.1136/annrheumdis-2017-eular.7212

\section{SP0030 BEYOND THE RECOMMENDATIONS: EXAMPLES OF SYSTEMATIC LITERATURE REVIEW IN DAILY CLINICAL PRACTICE}

K. Visser ${ }^{1,2} .{ }^{1}$ VU Medical Center; ${ }^{2}$ Academic Medical Center, Amsterdam, Netherlands

Recommendations and guidelines for management and treatment of rheumatic diseases exist to help rheumatologists deliver optimal care for their patients in an evidence-based way. However, not all practical questions and medical difficulties encountered by rheumatologists in daily clinical practice can be addressed using these existing guidelines. Moreover, time in practice is limited and usually does not allow for extensive systematic literature research to find evidence regarding a specific medical question that needs answering in due time. Therefore, an essential skill for doctors and those in training, besides medical knowledge and examination skills, is to be able to search for, withdraw and appraise published evidence applicable to a confined topic. A so called CAT - critically appraised topic - is a compact systematic literature research following a strictly formulated PICO to answer a clinical question encountered in daily practice. Results can be presented in department meetings and stored in a database for education purposes and use in daily practice. This presentation will address the structure, the carrying out, as well as important pros and cons of the CAT. Examples will be given drawn from the experience of young rheumatologists in training in the Netherlands.

Disclosure of Interest: None declared

DOI: 10.1136/annrheumdis-2017-eular.7307

\section{WEDNESDAY, 14 JUNE 2017}

\section{EULAR Campaign: Don't Delay, Connect Today}

\section{SP0031 HOW COULD GPS ENHANCE THE EARLY DIAGNOSIS OF RHEUMATIC DISEASES}

C.D. Mallen. Arthritis Research UK Primary Care Centre, Primary Care and Health Science, Keele, United Kingdom

Primary care and general practice has a key role to play in the early and accurate detection of patients with rheumatoid arthritis.

This talk will explore some of the current barriers to best practice, highlighting the workload associated with musculoskeletal problems in primary care and the challenges in making a prompt diagnosis. Possible solutions will be explored, highlighting the importance of strong patient and professional partnerships

Disclosure of Interest: None declared

DOI: 10.1136/annrheumdis-2017-eular.7239 